\title{
Refractometric biosensing based on optical phase flips in sparse and short-range-ordered nanoplasmonic layers
}

\begin{abstract}
Mikael Svedendahl, Ruggero Verre and Mikael Käll
Noble metal nanoparticles support localized surface plasmon resonances (LSPRs) that are extremely sensitive to the local dielectric properties of the environment within distances up to $10-100 \mathrm{~nm}$ from the metal surface. The significant overlap between the sensing volume of the nanoparticles and the size of biological macromolecules has made LSPR biosensing a key field for the application of plasmonics. Recent advancements in evaluating plasmonic refractometric sensors have suggested that the phase detection of light can surpass the sensitivity of standard intensity-based detection techniques. Here, we experimentally confirm that the phase of light can be used to precisely track local refractive index changes induced by biomolecular reactions, even for dilute and layers of short-range-ordered plasmonic nanoparticles. In particular, we demonstrate that the sensitivity can be enhanced by tuning in to a zero reflection condition, in which an abrupt phase flip of the reflected light is achieved. Using a cost-effective interference fringe tracking technique, we demonstrate that phase measurements yield an approximately one order of magnitude larger relative shift compared with traditional LSPR measurements for the model system of NeutrAvidin binding to biotinylated nanodisks.
\end{abstract}

Light: Science \& Applications (2014) 3, e220; doi:10.1038/lsa.2014.101; published online 7 November 2014

Keywords: biosensors; interferometry; metasurfaces; nanostructures; spectroscopy

\section{INTRODUCTION}

A considerable number of studies have demonstrated that localized surface plasmon resonances (LSPRs) in noble metal nanoparticles possess a number of promising characteristics for ultrasensitive and cost-effective label-free molecular analyses for the life sciences and beyond. ${ }^{1-4}$ Plasmons in metal nanoparticles can be used as transducers of biological and chemical interactions because the plasmon strongly couples to light and because its particle-specific resonance wavelength shifts if the concentration, composition or conformation of molecules changes within nanometric distances from the particle surface. ${ }^{1-4}$ Since the first report of an LSPR red-shift induced by molecular adsorption by Englebienne ${ }^{5}$ in 1998, colorimetric detection using classical optical spectroscopy methods has been the most popular transduction methodology. However, it has recently been proposed that the phase information contained in light scattered or reflected from nanoparticles excited at resonance could be used to implement alternative and even more effective LSPR sensing schemes. ${ }^{6-11}$ Indeed, phase interrogation techniques have significantly decreased the limits of detection in the case of classical surface plasmon resonance sensors based on planar gold films. ${ }^{7,12-14}$ In this case, the sensitivity enhancement is primarily due to the rapid phase flip that occurs when the reflectance approaches zero close to the resonance wavelength. Similar properties have been observed in studies of collective resonances in periodic arrays of nanoparticles, ${ }^{8,9}$ which experienced high bulk refractive index sensitivities due to sharp diffractive resonances in combination with zero reflection, or so-called 'topological darkness', a term coined by Kravets et al. ${ }^{8,15}$ However, large bulk refractive index sensitivities do not directly lead to high sensitivity to molecular adsorption in close proximity to the metal surface. Another important parameter is the spatial confinement of the refractive index sensitivity, as has been illustrated through comparisons between LSPRs in gold nanoparticles and propagating surface plasmons in thin gold films. ${ }^{16,17}$ Although the propagating plasmon has a bulk refractive index sensitivity that is almost twenty times greater than that of LSPRs, both methodologies yield similar resonance shifts upon molecular adsorption due to the better overlap between the sensitive fields and the molecules in the case of LSPRs. ${ }^{16}$

Although the collective resonances represent an interesting and promising route to extreme sensitivities, periodic arrays of nanoparticles with extremely narrow diffractive resonances require extreme precision during the fabrication process. The applicability of the phase-based methodology would clearly significantly increase if similar sensitivity could be achieved in less ordered structures, which are much simpler and less expensive to fabricate. From this perspective, the optical readout methodology can also be used to increase simplicity and decrease costs. However, there have only been a few reports on phase-based biosensing methodologies, ${ }^{8,10,11}$ and only one report utilized zero reflections (however, using collective resonances). ${ }^{8}$ To the best of our knowledge, no studies have performed biosensing using the zero reflection phase flip from purely localized plasmonic resonances.

Here, we show that the reflection from a sparse non-periodic layer of gold nanodisks bound to a glass/water interface can be used for phase-based refractometric biosensing in an inexpensive and simple interferometric measurement set-up. The sensitivity of the method is 
maximized by tuning the sample and measurement parameters to a zero reflection condition, in which the phase response is enhanced similar to the case of phase-based surface plasmon resonance sensing. The advantages of this method are compared to the established colorimetric detection technique, and the capability of the system is demonstrated through biorecognition between the $\sim 60 \mathrm{kD}$ protein NeutrAvidin (NA) and biotin bound to gold nanodisks.

This article is divided into two parts. We first provide experimental and theoretical guidelines on how to achieve zero reflection and the associated phase flip in a short-range-ordered plasmonic layer and discuss the influence of this condition on the phase of the reflected light. In the second part, we develop a spectrometer-free phase-based sensing scheme and discuss its advantages in comparison to normal colorimetric analysis.

\section{MATERIALS AND METHODS}

\section{Sample fabrication}

Poly(methyl methacrylate) was spin coated on top of a borosilicate glass slide, and then polystyrene beads were dispersed on the surface. After gold deposition, tape-stripping removal of the beads and etching of the poly(methyl methacrylate), the areas previously occupied by the beads were rendered available for the direct deposition of the desired material. Thus, the geometry of the resulting nanoparticles was primarily determined by the initial bead size and the amount of evaporated material. A 2-nm-thick layer of chromium was deposited underneath the gold to ensure proper adhesion. For laser diode measurements, we used a 5-mmthick glass substrate to decrease image distortions due to etalon effects.

\section{Optical spectroscopy}

A fiber-coupled halogen light source (HL-2000; Ocean Optics, Dunedin, FL, USA) was collimated and used as an illumination source. Spectra were collected using a fiber-coupled photodiode array spectrometer (BRC711E; B\&W Tek, Newark, DE, USA). Transmission and extinction spectra were collected with the illumination normal to the sample.

\section{Phase set-up}

The laser diode (HL6360MG; Oclaro, San Jose, CA, USA) wavelength was tuned to the minimum reflection wavelength through simple temperature adjustments of the casing. The two birefringent prisms (DPU25; Thorlabs, Newton, NJ, USA) were mounted together with their respective fast axes misaligned by $45^{\circ}$. Because of the wedge-like structure of the prisms, the retardation of a specific wavelength of light depends on the beam position on the double prisms, i.e., the retardation increases along a certain direction, $X$, perpendicular to the direction of propagation. Because the polarization states on the output side of the depolarizer vary with $X$, different polarization states illuminate different areas of the nanodisk array, generating spatially varying reflections. The reflections pass through a polarization analyzer that generates a fringe pattern due to the different polarization states reflected by the gold nanodisk layer. The fringes are captured on a color CCD (charge coupled device) chip (DC110; Thorlabs, Newton, NJ, USA). Note that the analyzer needs to be aligned such that it transmits similar amounts of $\mathrm{p}$ - and s-polarized light to obtain high contrast fringes that depend strongly on the phase difference between the two polarization components. Because the p-polarized reflectance is significantly larger than the s-polarized reflectance, the analyzer must be slightly detuned from transmitting pure s-polarized light.

\section{Biotin functionalization}

The gold nanoparticles were immersed in a 99\% thiol-PEG (NanoScience, Pheonix, AZ, USA) and 1\% thiol-EG-biotin (NanoScience) solution overnight. ${ }^{18}$ Afterwards, the layers were sonicated in pure (99\%) ethanol for $10 \mathrm{~min}$ to remove unbound or physisorbed thiols and then dried under a nitrogen atmosphere before being mounted in the flow cell. The NA (Sigma Aldrich, St Louis, MO, USA) experiment was performed in $0.01-\mathrm{mol} / \mathrm{L}$ phosphate buffer.

\section{RESULTS AND DISCUSSION}

\section{Zero reflections from a sparse and short-range-ordered plasmonic layer}

The condition of zero reflection can be realized for arrays of nanoparticles located at a dielectric interface by matching their scattered light intensity in the specular reflection angle to the direct reflection of the interface. We have previously reported that if the condition occurs at incidence angles larger than the critical angle, $\theta_{\mathbf{i}}>\theta_{\mathrm{c}}$, all incident light is absorbed by the nanoparticles. ${ }^{19}$ However, such constraints on $\theta_{\mathrm{i}}$ are not necessary to achieve rapid phase flips. Once the condition is satisfied, the phase associated with the specular reflection will vary rapidly with wavelength and angle across the resonance condition. This concept can be quantitatively illustrated using a model based on modified Fresnel coefficients. ${ }^{20-24}$ The reflection coefficients for s-polarized and p-polarized light, $r_{\mathrm{s}}$ and $r_{\mathrm{p}}$, can be written as follows: ${ }^{20,23,24}$

$$
\begin{gathered}
r_{\mathrm{s}}=\frac{n_{\mathrm{i}} \cos \theta_{\mathrm{i}}-n_{\mathrm{t}} \cos \theta_{\mathrm{t}}+i \frac{\omega}{c} \rho \alpha(\lambda)}{n_{\mathrm{i}} \cos \theta_{\mathrm{i}}+n_{\mathrm{t}} \cos \theta_{\mathrm{t}}-i \frac{\omega}{c} \rho \alpha(\lambda)} \\
r_{\mathrm{p}}=\frac{n_{\mathrm{t}} \cos \theta_{\mathrm{i}}-n_{\mathrm{i}} \cos \theta_{\mathrm{t}}-i \frac{\omega}{c} \rho \alpha(\omega) \cos \theta_{\mathrm{i}} \cos \theta_{\mathrm{t}}}{n_{\mathrm{t}} \cos \theta_{\mathrm{i}}+n_{\mathrm{i}} \cos \theta_{\mathrm{t}}-i \frac{\omega}{c} \rho \alpha(\omega) \cos \theta_{\mathrm{i}} \cos \theta_{\mathrm{t}}}
\end{gathered}
$$

where $\theta_{\mathrm{i}}$ and $\theta_{\mathrm{t}}$ are the angles of incidence and transmission, respectively, $\alpha(\lambda)$ is the wavelength-dependent polarizability parallel to the interface of a single nanoparticle and $\rho$ is the surface density of nanoparticles. We can study the variations in reflected intensity and phase as a function of the particle density by modeling the gold nanodisks as oblate spheroids located at a glass/water interface. Figure 1 shows that as $\rho$ decreases towards the zero reflection condition, which corresponds to the case when the numerator in Equation (1) approaches zero, the reflected phase exhibits an increasingly steeper transition. The rapid phase dispersion indicates that a small shift in the LSPR will result in a very large phase change for wavelengths near the reflectance minimum. This is the basic principle underlying phase interrogation methodologies for plasmonic biosensing applications.

Varying $\rho$ is one approach for tuning the reflection minima. As shown in Equation (1), the matching condition for achieving zero reflection depends on the refractive index contrast of the interface, $n_{\mathrm{i}}$ and $n_{\mathrm{t}}$; the incidence angle; and the product $\rho \alpha$. Therefore, rather than varying $\rho$, increasing or decreasing $\alpha$, by tuning the geometry or material of the nanoparticles may also lead to zero reflections. For a given $\rho \alpha$, on the other hand, the incidence angle needs to be fine-tuned to fulfill the condition. Naturally, the p-polarized reflections can also be minimized using Equation (2), as we have previously shown. ${ }^{19}$

Based on the theory presented in this work, we constructed samples that fulfilled the zero reflection condition for s-polarized light using hole-mask colloidal lithography. ${ }^{25}$ Figure $2 \mathrm{a}$ and $2 \mathrm{~b}$ shows the resulting gold nanoparticle layer as fabricated on an Si substrate to facilitate scanning electron microscopy imaging. The surface is decorated by gold nanodisks with a diameter and height of approximately 86 and $20 \mathrm{~nm}$, respectively, and the surface density is approximately 14 


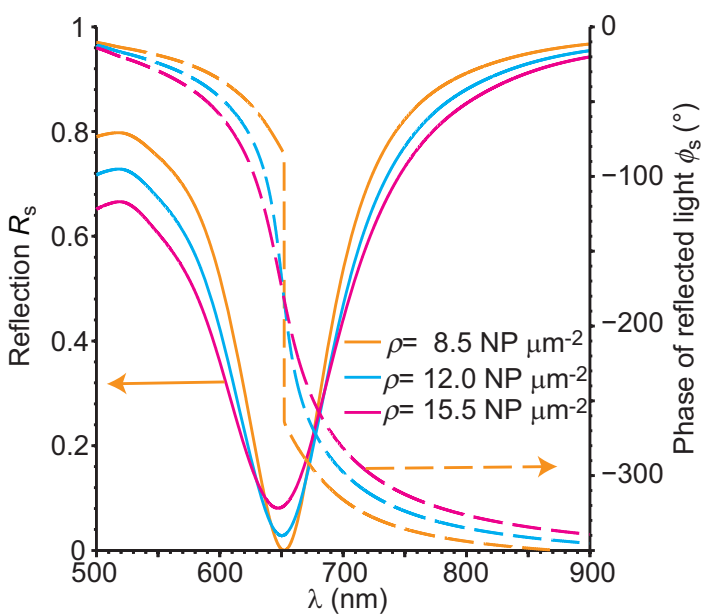

Figure 1 Illustration of the abrupt phase flip obtained from layers exhibiting zero reflection. The figure shows the reflected amplitude and phase for a layer of gold nanoparticles at various surface densities calculated using modified Fresnel coefficients for s-polarized light. The nanoparticles were simulated as oblate spheroids with minor and major axes of $20 \mathrm{~nm}$ and $80 \mathrm{~nm}$, respectively, located at a glass/water interface. The illumination angle corresponds to the critical angle of the glass/air interface. NP, nanoparticle.

nanodisks $\mu \mathrm{m}^{-2}$, or approximately $8 \%$ of the Si surface. Equivalent samples, which are shown in the photograph in Figure $2 c$, were fabricated on glass for optical characterization and sensing experiments. Hole-mask colloidal lithography is a robust, simple and inexpensive fabrication technique that can be used to produce homogeneous arrays on large scales. The samples shown in Figure $2 \mathrm{c}$ are one inch in diameter, and all of the samples possess nearly identical optical properties, as shown in Supplementary Fig. S1. Note that the particle density used is sufficiently high for the sample to effectively behave like a homogeneous medium, or a metamaterial, within the wavelength range of interest. This means that practically all reflected light is concentrated to the specular reflection angle and that diffuse scattering is negligible. ${ }^{16,19}$

Fine-tuning towards the zero reflection condition is easily achieved by altering the incidence illumination angle, compensating for small

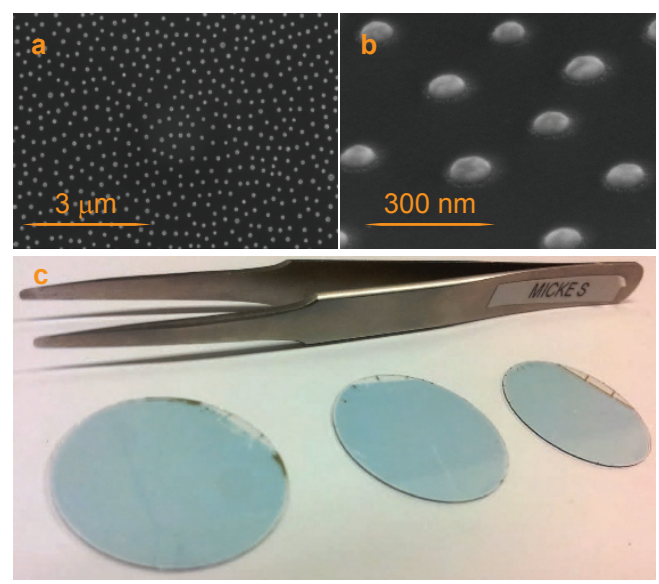

Figure 2 SEM images of typical gold nanodisk arrays and spectroscopic measurement of the sharp phase response near the zero reflection wavelength. (a and b) SEM and (c) photograph of one-inch homogeneous arrays of gold nanodisks with a mean diameter of $86 \mathrm{~nm}$ and height of approximately $20 \mathrm{~nm}$. SEM, scanning electron micrograph. deviations in the optical properties of the samples in Figure $2 \mathrm{c}$ in accordance with Equation (1). Figure 3 illustrates how the reflection can be tuned to minimize the reflection at a particular wavelength. Although all s-polarized spectra exhibit dips around $640-650 \mathrm{~nm}$, the reflection is as low as $1.5 \times 10^{-4} \pm 0.0001$ at $\theta_{\mathrm{i}}=55^{\circ}$ incidence, compared with 0.002 at $\theta_{\mathrm{i}}=50^{\circ}$ and $58^{\circ}$. As described in earlier reports, the reflection spectrum results from interference between the Lorentzianlike polarizability of the nanoparticles and the broad continuum of the bare interface reflection, which is glass/water in this case. ${ }^{19,20}$ Therefore, the reflection spectra can be visualized as a Fano resonance, with spectroscopic peaks, dips or asymmetric resonances depending on the relative strengths of the two interfering components. Thus, because $\mathrm{p}$-polarized and s-polarized reflections from the bare interface have different amplitudes within the incidence angle range investigated in Figure 3, the resulting spectra show peaks for p-polarized light and dips for s-polarized light.

By illuminating the layer with circularly polarized light through a glass prism and measuring different polarization states, the phase difference between p- and s-polarized reflections can be retrieved. Because we use circularly polarized light, the sample is illuminated by equal intensities of s- and p-polarized light. The s- and p-polarized reflection components were measured together with a component with the vibration vector oriented $45^{\circ}$ relative to the plane of incidence, which is denoted as $R_{\mathrm{s}+\mathrm{p}}$ in Figure 3. The phase can then be calculated from the relation as follows:

$$
\begin{aligned}
R_{\mathrm{s}+\mathrm{p}}(\lambda) & =\frac{\left|r_{\mathrm{p}}+i r_{\mathrm{s}}\right|^{2}}{2} \\
& =\frac{\left|r_{\mathrm{p}}(\lambda)\right|^{2}+\left|r_{\mathrm{s}}(\lambda)\right|^{2}+2\left|r_{\mathrm{p}}(\lambda)\right|\left|r_{\mathrm{s}}(\lambda)\right| \sin \Delta(\lambda)}{2}
\end{aligned}
$$

We modeled the optical response of the layer using the same methodology as in Refs. 19 and 20. In brief, the analytical polarizability of a gold ellipsoid is used together with the modified long wavelength approximation to represent the responses of individual nanoparticles. ${ }^{26-28}$ The only fitting parameter used was the effective refractive index surrounding the nanoparticles, located at an interface, which was tuned to match the resonance wavelength to an experimental transmission spectrum (Supplementary Fig. S2). The reflections were calculated using Equations (1) and (2) by inserting the model polarizability, the surface density of nanoparticles and the refractive index of the interface. The similarity between the experimental and theoretical data is striking, particularly considering that the only fitting parameter is the effective refractive index surrounding the nanoparticles.

As shown in Figure 4, we observed that $\Delta$ indeed varies faster as the reflection approaches zero. $\Delta$ shows the steepest response for $\theta_{\mathrm{i}}=55^{\circ}$, where the s-polarized reflection showed a minimum. The experimental data show that by properly adjusting the incidence angle by a few degrees, steeper phase responses can be achieved. The reflected phase shows a rapid $\sim 180^{\circ}$ flip as the zero reflection condition is traversed at $\theta_{\mathrm{i}}=55^{\circ}$. How fast the phase changes is dependent on both the incidence angle and on the wavelength, with the most rapid responses occurring close to the minimum reflection angle and wavelength. By tuning the incidence angle from $50^{\circ}$ to $55^{\circ}$, the phase response becomes steeper by a factor of 4.7 at the reflection minima. Because $\sin \Delta$, which is the actual measured parameter, is only defined between $\pm 90^{\circ}$ and because the uncertainty of the measurements is large around $\sin \Delta \approx \pm 1$ (as $\partial \sin \Delta \propto \partial \Delta \cos \Delta$ ), the recorded phase appears somewhat distorted compared with Figure 1. However, the 


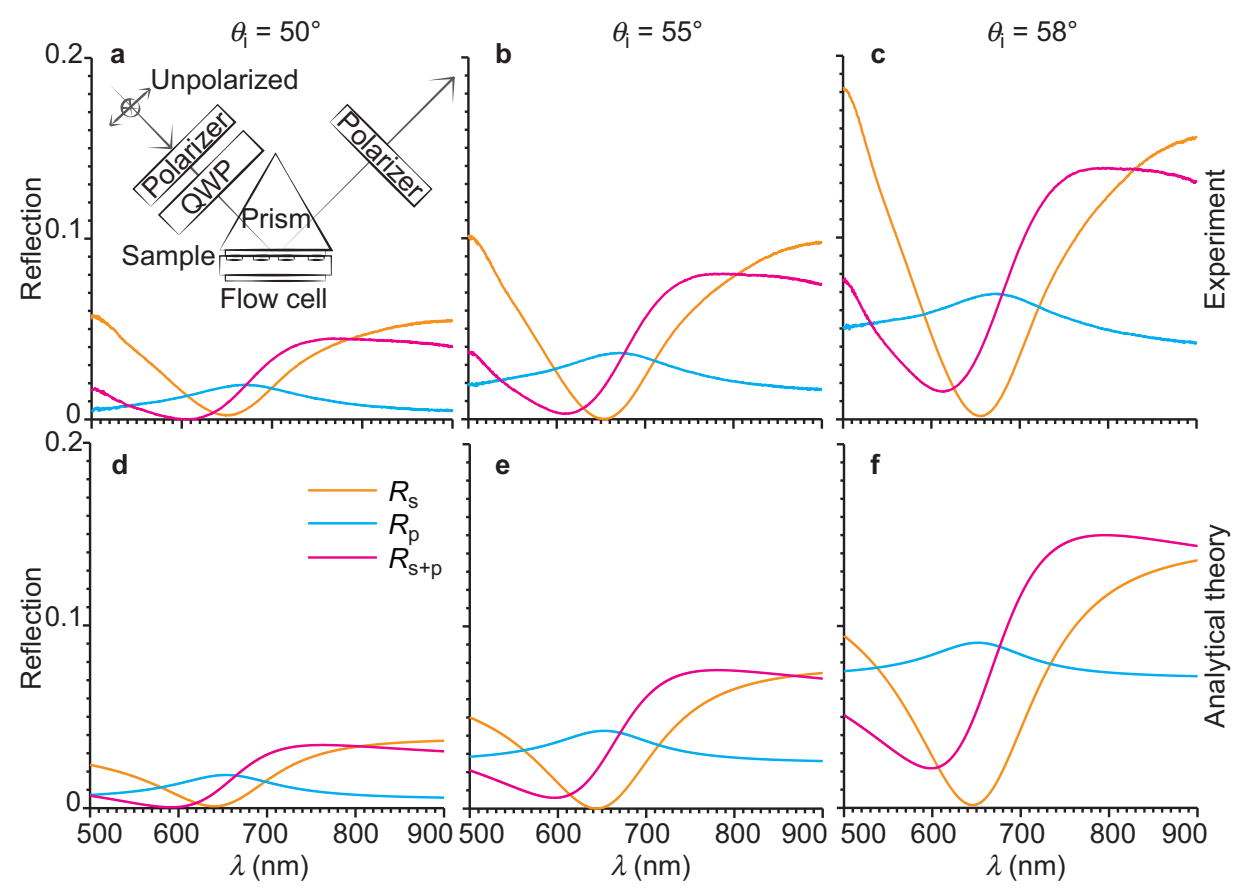

Figure 3 Reflection spectra for different incidence angles $\theta_{\mathrm{i}}$ and polarizations from a gold nanodisk layer in a water environment. The inset in (a) shows the experimental set-up, in which circularly polarized light is incident on the sample, and the reflection is measured at three different polarization states, with $R_{\mathrm{S}+\mathrm{p}}$ denoting a polarization state half-way between s-and p-polarization. (a-c) The experimental data show the deepest dip for a $55^{\circ}$ incidence and s-polarization. (d-f) With the exception of small deviations in intensity, the analytical theory, based on an ellipsoidal model of the nanoparticle's polarizability, captures the main characteristics of the reflections. QWP, quarter wave plate.

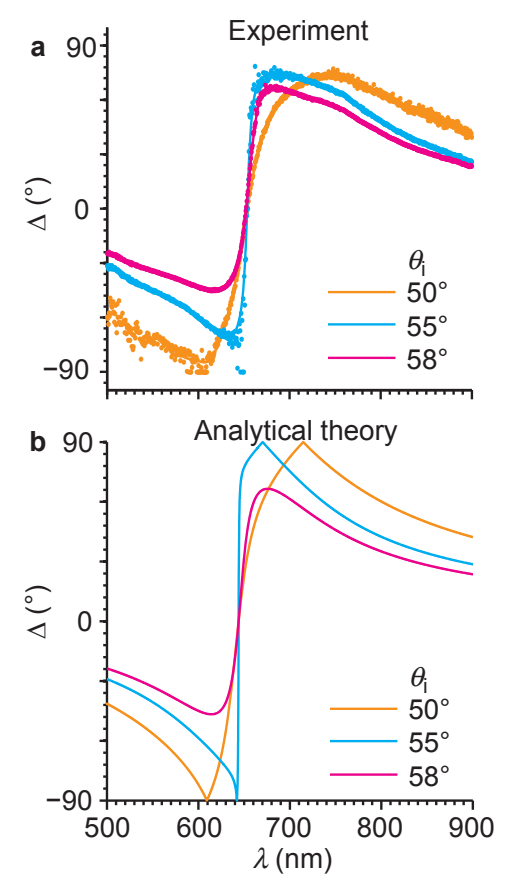

Figure 4 The phase difference between $\mathrm{p}$ - and s-polarized light, $\Delta$, calculated using the inversion of Equation (2) and the reflections in Figure 3. $\Delta$ varies the fastest at $\theta_{i}=55^{\circ}$, as expected from the minimized s-polarized reflection shown in Figure 3. Because $\sin \Delta$ is only defined in the range of $\pm 90^{\circ}$, some parts of the phase response are inverted. response does indeed agree well with analytical theory if $\Delta$ is extracted using Equation (3) with $R_{\mathrm{s}+\mathrm{p}}(\lambda)=\left|r_{\mathrm{p}}+i r_{\mathrm{s}}\right|^{2}$, where $r_{\mathrm{s}}$ and $r_{\mathrm{p}}$ are calculated from Equations (1) and (2), as illustrated in Figure $4 \mathrm{~b}$.

By mathematically adjusting the phase response for $55^{\circ}$ incidence (by inverting the phase values to the red of the singularities at $\pm 90^{\circ}$ ), a phase spectrum that appears more natural can be obtained, with a continuously varying function from approximately $0^{\circ}$ to $-360^{\circ}$, as shown in Figure 5 . The phase clearly varies much faster across the resonance position than the reflected intensity. This difference is clearly observed in Figure 5 a as the phase varies by approximately $14^{\circ}$ per nanometer in the vicinity of the reflection minimum, in comparison to less than $1^{\circ} \mathrm{nm}^{-1}$ calculated from $\alpha$ (Supplementary Fig. S3). Thus, there is a significant benefit in measuring phase responses in the vicinity of the zero reflection condition.

\section{Refractometric biosensing using phase interrogation}

Although the data in Figure 5 indicate that the spectroscopic measurement of $\sin \Delta$ is a promising sensing modality, a single wavelength detection scheme takes greater advantage of the spectral sharpness because it does not require expensive spectrometers and it can fully utilize the availability of inexpensive but powerful monochromatic light sources. As shown in Figure 6a, we implemented a simple interferometric phase interrogation scheme based on the work of Halpern et al. ${ }^{29,30}$ The set-up is based on off-the-shelf optical components and a laser diode emitting at $639 \mathrm{~nm}$, i.e., close to the reflection minimum of the nanoparticle layer. In brief, the collimated diode output is linearly polarized before passing through a double birefringent wedge prism that creates a spatially varying polarization state across the illuminated area. The nanoparticle sample is positioned on the backside of a borosilicate glass prism with an intermediate layer of index-matching oil. The reflected light passes through a polarization analyzer and 

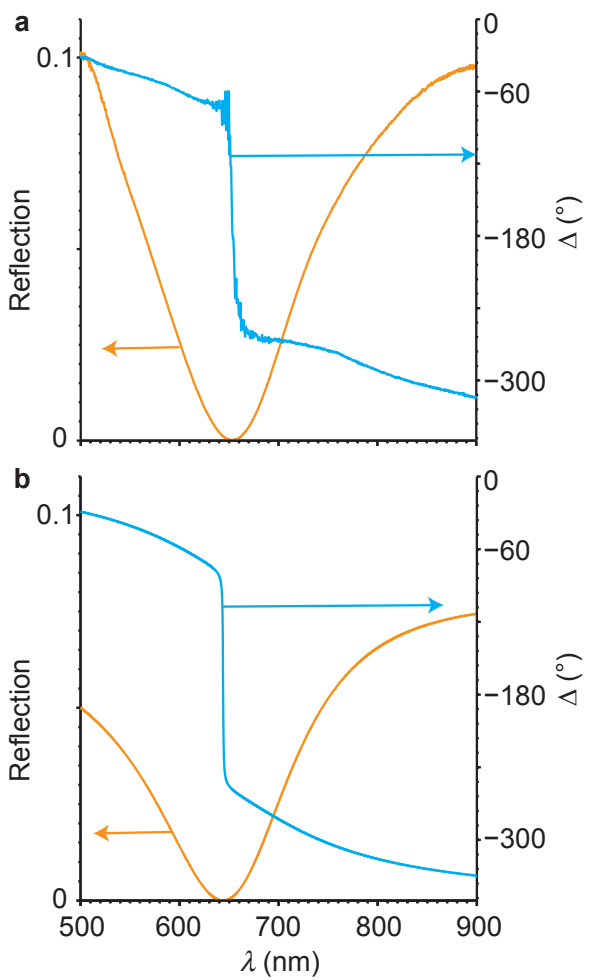

Figure 5 A spectral comparison between the phase response (blue) and the spolarized intensity response (orange). (a) Experimental and (b) theoretical phase and amplitude data at $\theta_{\mathrm{i}}=55^{\circ} . \Delta \mathrm{in}$ a is retrieved from the mathematical inversion of different regions in Figure $4 a$, whereas $\Delta$ in $\mathbf{b}$ is easily retrieved from the modified Fresnel formulas. generates a fringe pattern on a CCD chip due to interference between the $\mathrm{p}$ - and s-polarized reflection components. The resulting sinusoidal intensity variations, which are a direct measure of the change in the phase difference between $s$ and $p$, can then be tracked during the experiments.

The refractive index sensitivity of the nanoparticle layer was tested using the interferometric technique and compared to spectroscopic measurements performed in reflection and transmission modes. Solutions with different refractive indices were prepared by mixing water with various concentrations of ethylene glycol (EG). The shifts in the LSPR wavelength between pure water and solutions with $2.5 \%$ and $5 \%$ EG were on the order of $0.5 \mathrm{~nm}$ and $1 \mathrm{~nm}$, respectively (see Supplementary Information), which are in good agreement with previously reported bulk refractive index sensitivities for similar nanostructures. ${ }^{16,31,32}$

As illustrated in Figure 6b-6d, the corresponding fringe movements were approximately $50^{\circ}$ and $75^{\circ}$, respectively, for an analyzer angle of $4^{\circ}$. Note that the fringe shift obtained for the $2.5 \%$ EG solution relative to water is clearly observed by the naked eye (compare Figure $6 \mathrm{~b}$ and $6 \mathrm{c}$ ), whereas the colorimetric shift of $0.5 \mathrm{~nm}$ requires sensitive spectroscopic quantification to be observed. This result is a good indication of the intrinsic sensitivity of the interferometric detection methodology.

The fringe sensitivity does, however, depend on the alignment of the analyzer because it will transmit different ratios of $\mathrm{p}$ - and s-polarized light. Naturally, for an analyzer angle parallel to s-polarization, corresponding to $0^{\circ}$ in Figure $6 \mathrm{e}$, only s-polarized light is transmitted, and the fringes therefore do not shift upon changes in the refractive index. Because the s-polarized light reflected from the nanoparticle layer is considerably weaker than the reflected p-polarized light, larger analyzer angles are dominated by the p-polarized light and the interference
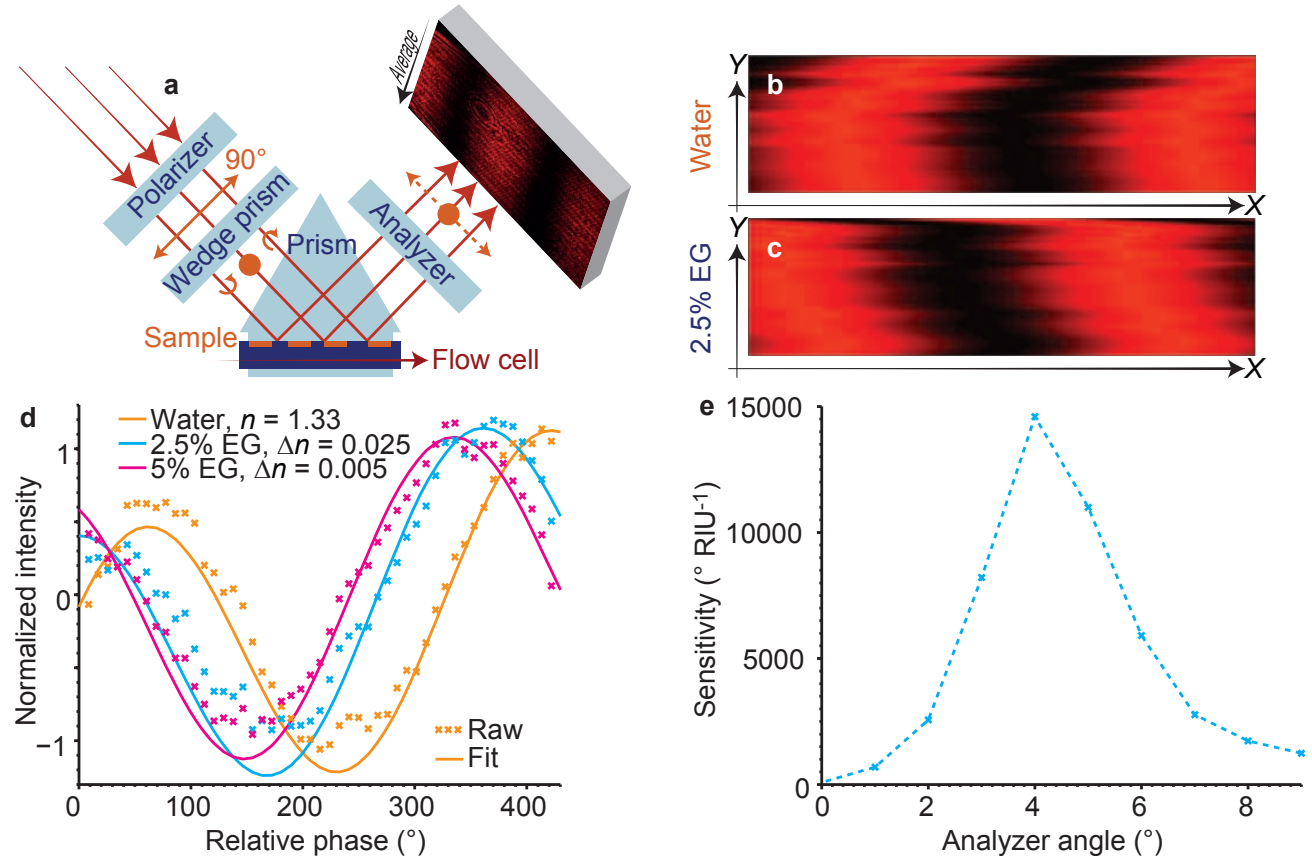

Figure 6 Bulk refractive index sensing with a single wavelength phase detection scheme. (a) The reflections from the nanoparticle layer were investigated using spatially varying polarization states of a collimated laser diode beam and analyzed using a polarizer, creating interference fringes from the interaction between the $p$ and s-polarized reflection components. (b-e) The fringes were detected using a color CCD, where the intensity was averaged along one axis $Y$ to form sinusoidal patterns. The fringes shifted when the ambient medium was changed from a pure water solution to $2.5 \%$ and $5 \%$ EG. In (d-e), the optimum analyzer angle exhibited a bulk refractive index sensitivity of approximately $15000^{\circ} \mathrm{RIU}^{-1}$. CCD, charge coupled device; EG, ethylene glycol. 
effect is weak. At the optimum analyzer angle, both components substantially contribute to the resulting fringe pattern.

To investigate the relevance of the interferometric methodology for bioassays, in which the plasmonic nanoparticles are typically functionalized with antibodies or similar capture agents linked to the metal surface, we studied the biorecognition reaction between NA and biotin. The experiment was performed in phosphate buffer with $100-\mu \mathrm{g} \mathrm{mL} \mathrm{m}^{-1}$ NA to facilitate the formation of a monolayer on the gold nanodisks. As shown in Figure $7 \mathrm{a}$, an $18.5^{\circ}$ shift of the fringes was measured after $35 \mathrm{~min}$. The corresponding colorimetric shift was $2.2 \mathrm{~nm}$ (Figure 7b).

Assuming that the molecular adsorption can be described as a random sequential adsorption process of non-interacting entities, a complete monolayer corresponds to $\sim 55 \%$ of the total nanoparticle surface area being covered by NA. ${ }^{33}$ The surface area of an individual nanoparticle is approximately $10000 \mathrm{~nm}^{2}$ and the molecules are approximately $5.6 \times 5.0 \times 4.0 \mathrm{~nm}^{3}$ in size, ${ }^{34}$ which corresponds to $\sim 200-275$ NA molecules per particle. Interestingly, the biomolecular sensitivity observed in this work is in good agreement with the data reported by Grigorenko and co-workers, who obtained a $\sim 25^{\circ}$ phase shift for $\sim 100$ streptavidin molecules per particle, despite the orders of magnitude difference in the bulk refractive index sensitivities $\left(\sim 15000^{\circ} \mathrm{RIU}^{-1}\right.$ in the present case compared with $\sim 100000^{\circ} \mathrm{RIU}^{-1}$ reported in Ref. 9). This contradiction can be explained by considering the different nanostructures investigated, that is, well-ordered particle arrays resulting in sharp diffractive resonances with considerably greater penetration depth into the bulk

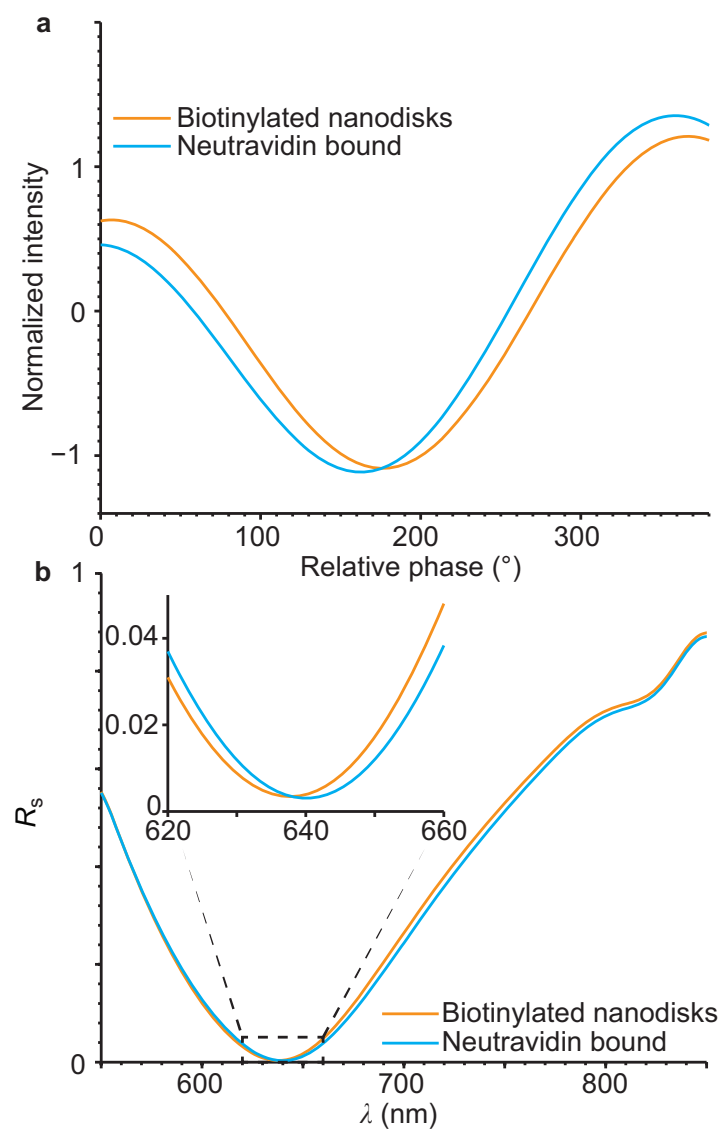

Figure 7 Refractometric sensing using fringe tracking and spectroscopy. (a) Tracking the minimum fringe position during the binding of a monolayer of NeutrAvidin to biotinylated gold nanodisks resulted in a phase shift of $18.5^{\circ}$ and (b) a shift of the $R_{\mathrm{s}}$ minimum wavelength by $2.2 \mathrm{~nm}$. environment compared to the samples studied here, for which shortrange optical near-fields dominate the response. Additionally, the holemask colloidal lithography fabrication technique is significantly less expensive, faster and simpler than the electron beam lithography-based fabrication used in Refs. 8 and 9.

To quantitatively compare the performances of the interferometric and spectroscopic detection schemes, we can define a visibility measure by normalizing the adsorption-induced shifts to the full-width at half-maximum of the fringe/spectral resonance. Using this approach, the visibilities of the phase shifts are approximately one order of magnitude greater than that for regular colorimetric detection. The fringe shift that occurs upon NA adsorption corresponds to $\sim 10.6 \%$ of the full-width at half-maximum, whereas the dip in the reflection spectrum shifts by $\sim 2.2 \mathrm{~nm}$ (Figure $7 \mathrm{~b}$ ), which is $\sim 1.4 \%$ of the spectral width. However, note that many bioassays rely on measurements of the kinetics of molecular interactions rather than of the final, endpoint, response of the sensor. We found that the performance in terms of stability over time was still better for the more mature spectroscopic technique: tracking the fringes over time resulted in a standard deviation of $\sim 0.01^{\circ}$ during $100 \mathrm{~min}$ (see the Supplementary Information), and the spectroscopic peak position only fluctuated by $0.5 \mathrm{pm}$. The signal-to-noise ratio in terms of the shift relative to temporal fluctuations is then $18.5 \% .01^{\circ}=1850$ compared with $2.2 \mathrm{~nm} / 0.0005 \mathrm{~nm}=4400$ for the phase and spectroscopic measurements, respectively. The discrepancy is at least partly due to stability issues of the rather inexpensive light source and detector used in the fringe set-up, which costs approximately one-tenth of the equipment required for the colorimetric detection scheme. Substantial performance improvements can most likely be achieved by further optimizing the set-up and equipment.

\section{CONCLUSIONS}

In summary, we have shown how plasmonic metamaterial layers that consist of sparse and short-range-ordered arrays of gold nanodisks may be designed to achieve a zero reflection condition, and we have demonstrated how to use the abrupt optical phase flip that is a consequence of this phenomenon for biosensing applications. By finetuning the incidence angle, the particle surface density and the oscillator strength of the particles, we obtained a plasmonic metasurface with a large phase dispersion suitable for interferometric analysis. A simple optical interferometry set-up was constructed, and measurements of bulk refractive index sensing and biosensing were performed and compared to standard colorimetric analysis. The results demonstrated that the obtained phase shifts had superior visibility compared to the spectroscopic shifts.

There are naturally a number of possible improvements to the presented methodology that may lead to substantially better signal-tonoise ratios and sensitivities. These improvements include obvious technical improvements, such as better isolation of the optical setup from temperature fluctuations and vibrations; better sample properties, such as higher particle homogeneity; and improvements to the measurement scheme. Note that the optical set-up shown in Figure 6 has the drawback that different areas of the nanoparticle layer are illuminated with different polarization states. Variations in sample uniformity, particularly the nanoparticle surface density, may subsequently decrease the quality of the fringe pattern and limit the minimum reflectance from the layer. The spectroscopic technique, on the other hand, integrates over the entire illuminated area, which should result in a more efficient ensemble averaging. It is thus possible that alternative phase-sensitive methodologies, for instance, based on 
phase-stepping or wave-plate rotation techniques, which also measure from the entire illuminated area, could increase the signal-to-noise ratio of the measurements. Similarly, a more uniform array could, in principle, decrease the minimum reflection amplitude even further and thus, increase the sensitivity of the phase measurements. It is hoped that further optimization along these lines will lead to costeffective sensing devices suitable for a wide range of biological and chemical analysis applications.

\section{ACKNOWLEDGEMENTS}

This work was financially supported by Vinnova, by the Swedish Foundation for Strategic Research and by the Knut and Alice Wallenberg Foundation.

1 Anker JN, Hall WP, Lyandres O, Shah NC, Zhao J et al. Biosensing with plasmonic nanosensors. Nat Mater 2008; 7: 442-453.

2 Zijlstra P, Paulo PM, Orrit M. Optical detection of single non-absorbing molecules using the surface plasmon resonance of a gold nanorod. Nat Nanotechnol 2012; 7 379-382.

3 Brolo AG. Plasmonics for future biosensors. Nat Photonics 2012; 6: 709-713.

4 Larsson EM, Langhammer C, Zorić I, Kasemo B. Nanoplasmonic probes of catalytic reactions. Science 2009; 326: 1091-1094.

5 Englebienne P. Use of colloidal gold surface plasmon resonance peak shift to infer affinity constants from the interactions between protein antigens and antibodies specific for single or multiple epitopes. Analyst 1998; 123: 1599-1603.

6 Lodewijks K, Van Roy W, Borghs G, Lagae L, Van Dorpe P. Boosting the figure-of-merit of LSPR-based refractive index sensing by phase-sensitive measurements. Nano Lett 2012; 12: 1655-1659.

7 Huang YH, Ho HP, Kong SK, Kabashin AV. Phase-sensitive surface plasmon resonance biosensors: methodology, instrumentation and applications. Ann Phys 2012; 524: 637-662.

8 Kravets VG, Schedin F, Jalil R, Britnell L, Gorbachev RV et al. Singular phase nanooptics in plasmonic metamaterials for label-free single-molecule detection. Nat Mater 2013; 12: 304-309.

9 Kravets VG, Schedin F, Kabashin AV, Grigorenko AN. Sensitivity of collective plasmon modes of gold nanoresonators to local environment. Opt Lett 2010; 35: 956-958.

10 Sannomiya T, Balmer TE, Hafner C, Heuberger M, Voros J. Optical sensing and determination of complex reflection coefficients of plasmonic structures using transmission interferometric plasmonic sensor. Rev Sci Instrum 2010; 81: 053102.

11 Moirangthem RS, Chang YC, Wei PK. Investigation of surface plasmon biosensing using gold nanoparticles enhanced ellipsometry. Opt Lett 2011; 36: 775-777.

12 Li YC, Chang YF, Su LC, Chou C. Differential-phase surface plasmon resonance biosensor. Anal Chem 2008; 80: 5590-5595.

13 Nelson SG, Johnston KS, Yee SS. High sensitivity surface plasmon resonance sensor based on phase detection. Sens Actuators B 1996; 35-36: 187-191.

14 Kabashin AV, Patskovsky S, Grigorenko AN. Phase and amplitude sensitivities in surface plasmon resonance bio and chemical sensing. Opt Express 2009; 17: 21191-21204.

15 Malassis L, Massé P, Tréguer-Delapierre M, Mornet S, Weisbecker P et al. Topological darkness in self-assembled plasmonic metamaterials. Adv Mater 2014; 26: 324 330.
16 Svedendahl M, Chen S, Dmitriev A, Käll M. Refractometric sensing using propagating versus localized surface plasmons: a direct comparison. Nano Lett 2009; 9: 44284433.

17 Yonzon CR, Jeoungf E, Zou SL, Schatz GC, Mrksich M et al. A comparative analysis of localized and propagating surface plasmon resonance sensors: the binding of concanavalin A to a monosaccharide functionalized self-assembled monolayer. J Am Chem Soc 2004; 126: 12669-12676.

18 Chen S, Svedendahl M, Antosiewicz TJ, Käll M. Plasmon-enhanced enzyme-linked immunosorbent assay on large arrays of individual particles made by electron beam lithography. ACS Nano 2013; 7: 8824-8832.

19 Svedendahl M, Johansson P, Käll M. Complete light Annihilation in an ultrathin layer of gold nanoparticles. Nano Lett 2013; 13: 3053-3058.

20 Svedendahl M, Käll M. Fano interference between localized plasmons and interface reflections. ACS Nano 2012; 6: 7533-7539.

21 Otte MA, Estévez MC, Regatos D, Lechuga LM, Sepúlveda B. Guiding light in monolayers of sparse and random plasmonic meta-atoms. ACS Nano 2011; 5: 9179-9186.

22 Thongrattanasiri S, Koppens FH, García de Abajo FJ. Complete optical absorption in periodically patterned graphene. Phys Rev Lett 2012; 108: 047401.

23 Bedeaux D, Vlieger J. Optical properties of surfaces. London: Imperial Collage Press, 2001.

24 Mendoza-Galván A, Järrendahl K, Dmitriev A, Pakizeh T, Käll M et al. Optical response of supported gold nanodisks. Opt Express 2011; 19: 12093-12107.

25 Fredriksson H, Alaverdyan Y, Dmitriev A, Langhammer C, Sutherland DS et al. Holemask colloidal lithography. Adv Mater 2007; 19: 4297-4302.

26 Yang WH, Schatz GC, Van Duyne RP. Discrete dipole approximation for calculating extinction and Raman intensities for small particles with arbitrary shapes. J Chem Phys 1995; 103: 869-875.

27 Zeman EJ, Schatz GC. An accurate electromagnetic theory study of surface enhancement factors for $\mathrm{Ag}, \mathrm{Au}, \mathrm{Cu}, \mathrm{Li}, \mathrm{Na}, \mathrm{Al}, \mathrm{Ga}, \mathrm{In}, \mathrm{Zn}$, and $\mathrm{Cd}$. J Phys Chem 1987; 91: 634-643.

28 Zorić I, Zäch M, Kasemo B, Langhammer C. Gold, platinum, and aluminum nanodisk plasmons: material independence, subradiance, and damping mechanisms. ACS Nano 2011; 5: 2535-2546.

29 Halpern AR, Chen YL, Corn RM, Kim D. Surface plasmon resonance phase imaging measurements of patterned mono layers and DNA adsorption onto microarrays. Anal Chem 2011; 83: 2801-2806.

30 Zhou WJ, Halpern AR, Seefeld TH, Corn RM. Near infrared surface plasmon resonance phase imaging and nanoparticle-enhanced surface plasmon resonance phase imaging for ultrasensitive protein and DNA biosensing with oligonucleotide and aptamer microarrays. Anal Chem 2012; 84: 440-445.

31 Dahlin AB, Tegenfeldt JO, Höök F. Improving the instrumental resolution of sensors based on localized surface plasmon resonance. Anal Chem 2006; 78: 4416-4423.

32 Chen S, Svedendahl M, Käll M, Gunnarsson L, Dmitriev A. Ultrahigh sensitivity made simple: nanoplasmonic label-free biosensing with an extremely low limit-of-detection for bacterial and cancer diagnostics. Nanotechnology 2009; 20: 434015.

33 Adamczyk Z, Siwek B, Zembala M, Belouschek P. Kinetics of localized adsorption of colloid particles. Adv Colloid Interface Sci 1994; 48: 151-280.

34 Rosano C, Arosio P, Bolognesi M. The X-ray three-dimensional structure of avidin. Biomol Eng 1999; 16: 5-12.

\footnotetext{
(c) (i) $(-)$ This work is licensed under a Creative Commons Attribution(c) $(1)\left({ }_{\mathrm{BY}} \mathrm{NC}\right.$ ND NonCommercial-NoDerivs 3.0 Unported License. The images or other third party material in this article are included in the article's Creative Commons license, unless indicated otherwise in the credit line; if the material is not included under the Creative Commons license, users will need to obtain permission from the license holder to reproduce the material. To view a copy of this license, visit http://creativecommons.org/licenses/ by-nc-nd/3.0/
} 